\title{
A Girl Who is Smiling for no Reason: Gelastic Seizure- A Case Report
}

\section{Subodh Sharma Paudel ${ }^{1}$ and Nikhil Agrawal ${ }^{2}$}

${ }^{1}$ Upendra Devkota Memorial National Institute of Neurological and Allied Sciences, Bansbari, Kathmandu,Nepal

${ }^{2}$ Nepalese Army Institute of Health Sciences, Shree Birendra Hospital, Chhauni, Kathmandu, Nepal

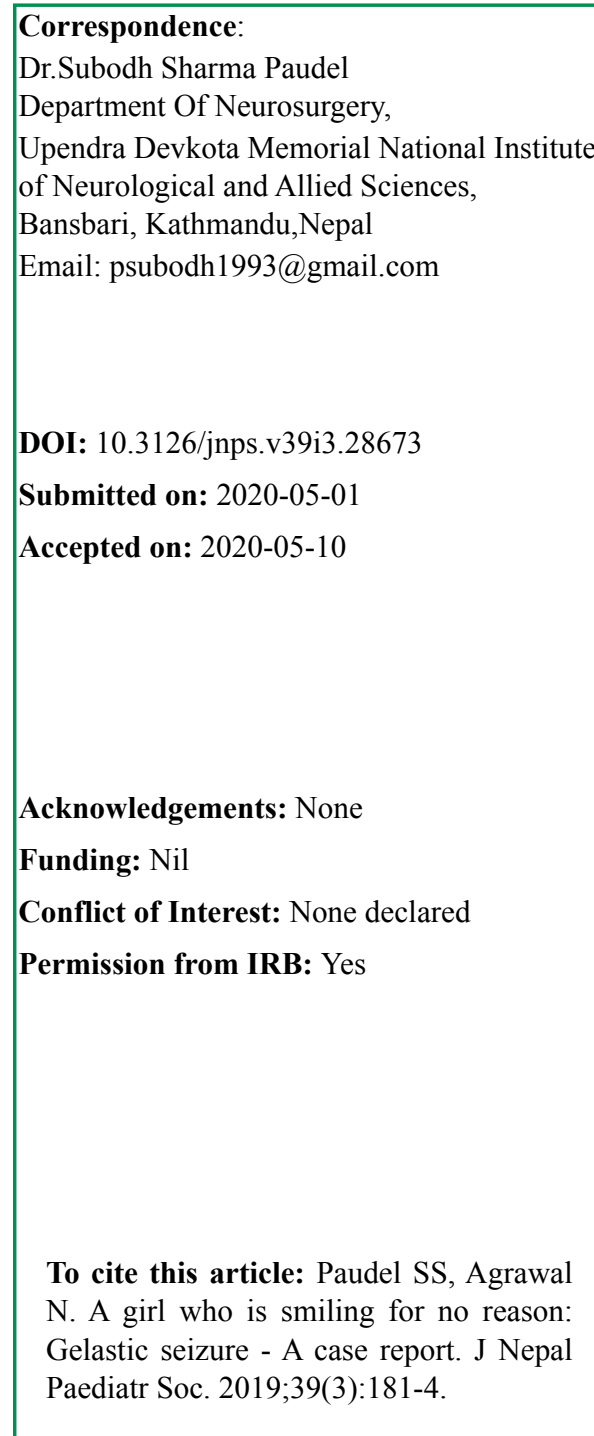

\section{ABSTRACT}

Being a rare disorder, Gelastic seizure primarily presents as episodes of sudden occurrence of emotions usually associated with laugh or giggle due to facial contractions. A child presented with abnormal body movement in the form of smiling and uprolling of eyes, which was clinically diagnosed as gelastic seizure, a rare form of a very common presentation, epilepsy. The case is presented to highlight abnormal posturing and smiling episodes in a child can be a form of seizure episode, which generally presents with a diagnostic dilemma and therapeutic nightmare to the clinician.
\end{abstract}

Keywords: functional brain imaging; Gelastic epilepsy; hypothalamic hamartoma; smiling attitude; Video-EEG

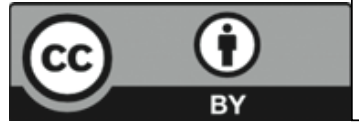

This work is licensed under creative common attribution 3.0 license 


\section{INTRODUCTION}

Gelastic seizure is a rare form of seizure disorder that primarily presents as episodes of sudden occurrence of emotions usually associated with laugh or giggle due to facial contractions. ${ }^{1}$ The most common cause of gelastic seizure is tumour of hypothalamus which may be hamartoma or astrocytoma. If any exact focal localisation of seizure is not found, it is categorised as cryptogenic localisation related epilepsy (LRE). ${ }^{2}$ We present a case of gelastic seizure who had presented to us with bouts of laughter events.

\section{CASE REPORT}

A four years old girl with no significant past history presented to our OPD with complaints of abnormal movements of upper and lower limbs, up-rolling of eyes and abnormal smiling expression for more than one month. This lasted for a minimum of five seconds and maximum up to two minutes with an average of eight to 10 episodes per day, associated with loss of consciousness. She had postictal confusional state lasting for about 30 seconds to one minute. It was not associated with fever or other triggers. It was occurring on daily basis, commonly during early mornings and evenings. Instead of seeking medical advice, she was taken for traditional modes of treatment, which was of no avail to her ailment. On examination, she was alert, active and oriented with normal anthropometry, general and systemic examination. Her ophthalmologic and otolaryngological evaluation was normal.

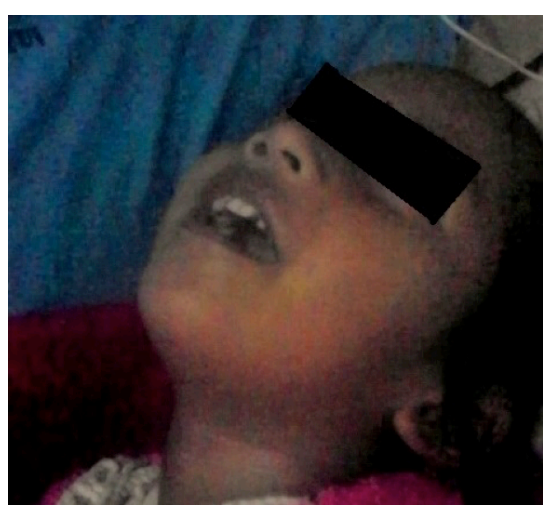

Figure 1. Smiling attitude in four old child during seizure episode
The provisional diagnosis of seizure disorder with smiling episodes was made. All her blood investigations were within normal limits. Computed tomography scan was also normal. It was done to rule out neurocysticercosis (NCC) and calcified granulomas. Magnetic resonance imaging (MRI) was done to look for presence of hypothalamic hamartoma, astrocytoma or temporal lobe sclerosis. MRI revealed no such pathology. Lumbar puncture was also normal. Electroencephalogram (EEG) was performed which showed background activity consisting of 5-6 Hz and generalised sharp and slow waves lasting for more than 10 seconds. (figure 1 )

She was started on sodium valproate and carbamazepine which showed no response. She was then added on with levetiracetam. She showed gradual improvement with total of one to two episodes lasting for about three to five seconds. Awake electro-encephalogram was performed which showed centro-temporal epilepsy with secondary generalisation. Thus the final diagnosis of clinical gelastic epilepsy was made. At present, she is on multiple anti-epileptic medications. The patient's parents were counselled about the cryptogenic nature of the disorder and its guarded prognosis.

\section{DISCUSSION}

The term gelastic epilepsy was coined by Daly and Mulder in 1957, meaning "gelos" (laughter) in Greek. ${ }^{1,3}$ Gascon and Lombroso (1971) proposed that gelastic epilepsy is diagnosed on the basis of stereotyped recurrence, absence of external

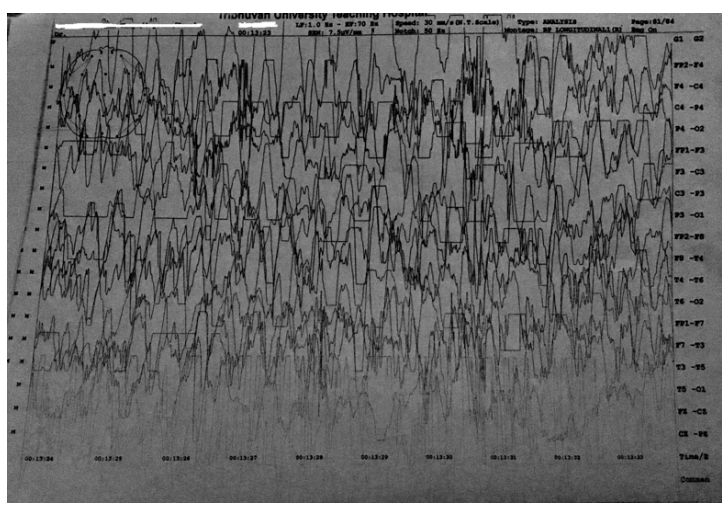

Figure 2. Ictal EEG of a girl four and half years of age with intact neurological, physiological development and frequent Gelastic seizure episodes 
precipitants, concomitant manifestations accepted as epileptic, presence of interictal electroencephalography (EEG) abnormalities and absence of conditions that can cause pathologic laughter. $^{4}$

Presently gelastic epilepsy is classified under Epilepsy Syndrome as a focal seizure with a structural abnormality. But, no recent guideline has been developed for evaluation of gelastic epilepsy. The laughter may be silent, a facial expression of smile or loud with natural vocalisations at various intensity and combination. There is no emotional element of pleasure or amusement associated with this: it is a mirthless laughter. ${ }^{5}$ The childhood prevalence of hypothalamic hamartoma with gelastic seizures is around 0.5 per 100,000 . There are no estimates of the prevalence of gelastic seizures in patients without hypothalamic hamartoma. The number of cases reported in the literature is considerably smaller. ${ }^{5}$

The smile is produced by various facial movements (buccal, palpebral and ocular) which are most commonly clonic in nature. It may or may not have secondary generalisations. There is gradual change in seizure pattern with age. The frequency and intensity at which seizure occurs may remain static or may show change in expression. ${ }^{6}$ Intrinsic epileptic capacity of hypothalamic hamartoma leading to gelastic seizure is attributed to two groups of pyramidal neurons. The small pyramidal neurons fire spontaneously expressing GABA (Gamma-Aminobutyric acid) receptors and large pyramidal neurons hyperpolarise in response to GABA. This synchronous firing of action potential leads to development of gelastic seizure. ${ }^{7}$

Gelastic episodes may be confused with unexplained crying of infancy or infantile colic. Similar type of pathologic laughter may be present in other conditions of nervous system like progressive supranuclear palsy, multiple sclerosis, amyotropic lateral sclerosis, tuberous sclerosis, focal obstructive hydrocephalus, focal cortical dysplasia, psychiatric illness, gelastic cataplexy with Niemann Pick disease type C etc. ${ }^{6,10}$ The diagnosis between these conditions and gelastic seizure is primarily made on the basis of Gascon and Lombroso (1971) criteria and videoelectroencephalography monitoring and use of high resolution MRI. ${ }^{4}$

EEG monitoring can be divided into ictal and interictal. Inter-ictal EEG is generally non informative and shows focal slowing and epileptiform activity and ictal EEG shows bilaterally synchronous and generalised epileptiform activity along with low voltage episodic fast rhythm with suppression of episodic background activity. ${ }^{8}$ In our case EEG showed generalised sharp and slow waves lasting $>$ 10 secs and right centro- temporal epilepsy with secondary generalization. It is mandatory to perform MRI of brain with an appropriate high resolution epilepsy imaging protocol in patients with gelastic seizures

Gelastic seizure is generally highly resistant to medical therapy. Some anti epileptic drugs when used in combination like carbamazepine, clobazam and vigabatrin, levetiracetam can produce slight decrease in frequency and duration of seizure but total remission of seizure activity is not obtained. It was found that the response of patients towards sodium valproate at relatively higher dose and carbamazepine produced better result. Generally, polytherapy does more harm than good. ${ }^{9}$ If space occupying lesion or hypothalamic lesions are found, surgical removal of those lesions offers complete remission of symptoms but non-surgical procedure like radiosurgery is gaining popularity because of better results. ${ }^{?}$

\section{CONCLUSIONS}

Gelastic seizure is a rare disorder which presents with atypical symptoms like laughing or giggling. It requires accurate history, observation, and vast investigations to diagnose it. It has to be differentiated from other organic and psychiatric disorders. It is generally refractory to anticonvulsant therapy. Intracranial pathology must be looked for possibility of surgical resection, which would lead to complete remission of the disease. 


\section{REFERENCES}

1. Uribe-San-Martin R, Ciampi E, Lawson-Peralta B, Acevedo-Gallinato K, Torrealba-Marchant G, Campos-Puebla M et al. Gelastic epilepsy: Beyond hypothalamic hamartomas. Epilepsy Behav Case Rep. 2015;4:70-3. DOI:10.1016/ j.ebcr.2015.07.001

2. Abou-Khalil B. Hypothalamic Hamartomas - What Determines Seizure Types and Other Clinical Manifestations? Epilepsy Curr. 2012;12(2):59-60. DOI: 10.5698/1535-7511-12.2.59

3. Pearce J. A Note on Gelastic Epilepsy. Eur Neurol. 2004;52(3):172-4. DOI: 10.1159/000081858

4. Chaudhry N, Puri V, Patidar Y, Khwaja G. Pathological laughter associated with paroxysmal kinesigenic dyskinesia: A rare presentation of acute disseminated encephalomyelitis. Epilepsy Behav Case Rep. 2013;1:14-9. DOI: 10.1016/j.ebcr.2012.11.001

5. Scheffer I, Berkovic S, Capovilla G, Connolly M, French J, Guilhoto L, et al. ILAE classification of the epilepsies: Position paper of the ILAE Commission for Classification and Terminology. Epilepsia. 2017;58(4):512-21. DOI: 10.1111/epi.13709

6. Panayiotopoulos C. A Clinical Guide to Epileptic Syndromes and their Treatment. 2nd ed. Dordrecht: Springer; 2010. Page 317-320.

7. Engel.Jr J, A. Pedley T. Epilepsy a comprehensive textbook. 2nd ed. Philadelphia: Wolters Kluwer; 2008. Page 619-622

8. Tellez-Zenteno J, Serrano-Almeida C, Moien-Afshari F. Gelastic seizures associated with hypothalamic hamartomas. An update in the clinical presentation, diagnosis and treatment. Neuropsychiatr Dis Treat. 2008:1021. DOI: $10.2147 /$ ndt.s2173

9. Harvey A, Freeman J. Epilepsy in Hypothalamic Hamartoma: Clinical and EEG Features. Semin Pediatr Neurol. 2007;14(2):60-4. DOI:10.1016/j.spen.2007.03.003

10. Panagariya A, Sharma B, Tripathi G, Kumar H, Agarwal V. Gelastic epilepsy associated with lesions other than hypothalamic hamartoma. Ann Indian Acad Neurol. 2007;10:1058. DOI: 10.4103/0972-2327.33218 